\title{
ChemComm
}

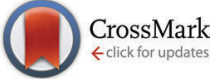

Cite this: Chem. Commun., 2017 53,2279

Received 17th December 2016 Accepted 25th January 2017

DOI: $10.1039 / \mathrm{c} 6 \mathrm{cc} 10046 \mathrm{e}$

rsc.li/chemcomm

\section{Controlling and tuning the dynamic nature of supramolecular polymers in aqueous solutions $\uparrow$}

\author{
Simone I. S. Hendrikse, ${ }^{a}$ Sjors P. W. Wijnands, ${ }^{a}$ René P. M. Lafleur, ${ }^{a}$ \\ Maarten J. Pouderoijen, ${ }^{\mathrm{b}}$ Henk M. Janssen, ${ }^{\mathrm{b}}$ Patricia Y. W. Dankers ${ }^{\mathrm{a}}$ and \\ E. W. Meijer*a
}

\begin{abstract}
Structural and kinetic exchange properties of supramolecular polymers composed of mono- and bivalent ureidopyrimidinone-based monomers are investigated in aqueous solutions. It is shown that exchange dynamics can be controlled by mixing different types of monomers. This tunability widens the scope in their design as biomaterials.
\end{abstract}

Water-soluble supramolecular polymers constitute an attractive class of polymers, since the non-covalent interactions between the molecular components can result in tuneable hydrogels with a highly dynamic structure. ${ }^{1-3}$ When applied as a biomaterial, the dynamic, adaptable, and reversible interactions between the hydrogel and embedded (stem) cells are proposed to closely mimic systems and processes found in nature. The artificial biomaterial developed shall not only contain specific mechanical properties, but also has to present proteins and peptides to cells in a reversible, adaptive and spatiotemporal manner in order to regulate cell response. ${ }^{4,5}$ Therefore, control over the structural and dynamic properties of hydrogels is important for the design and development of supramolecular biomaterials, which can only be achieved when there is profound understanding at the molecular level.

Many techniques are currently used to study such supramolecular systems in aqueous solutions. ${ }^{6}$ Förster resonance energy transfer (FRET) experiments ${ }^{7,8}$ have been used to study exchange dynamics in a broad spectrum of systems from liposomes ${ }^{9}$ to polymers. In addition, fluorescence microscopy, and more recently super resolution microscopy techniques such as stochastic optical reconstruction microscopy (STORM) yield valuable structural and temporal information of systems ranging from giant unilamellar vesicles to $1 \mathrm{D}$-supramolecular

\footnotetext{
${ }^{a}$ Institute for Complex Molecular Systems, Eindhoven University of Technology, P.O. Box 513, 5600 MB Eindhoven, The Netherlands. E-mail: e.w.meijer@tue.nl; Tel: +310402473101

${ }^{b}$ SyMO-Chem B.V., Het Kraneveld 14, 5612 AZ Eindhoven, The Netherlands $\dagger$ Electronic supplementary information (ESI) available: Synthetic procedures, methods, and supporting data of cryo-TEM, FRET, STORM and UV-vis experiments. See DOI: $10.1039 / \mathrm{c} 6 \mathrm{cc} 10046 \mathrm{e}$
}

aggregates such as benzene-1,3,5-tricarboxamide based polymers and peptide amphiphiles. ${ }^{10-12}$ Furthermore, stimulated emission depletion (STED) microscopy has been successfully applied in studying the formation and characteristics of multicomponent self-sorted supramolecular hydrogels in real-time. ${ }^{13}$

Using molecular design parameters to tune the macroscopic properties of hydrogels has been nicely shown by Scherman and Stupp. Scherman has shown that different guests for cucurbit[8]uril mediated crosslinking resulted in distinct crosslink dynamics, influencing the mechanical properties at the hydrogel level. ${ }^{14}$ Stupp has revealed that a disordered fibre packing can be obtained by substituting valine for alanine in peptide amphiphiles, which subsequently decreases the physical properties of the hydrogel. ${ }^{15}$

Here we have investigated the structural and kinetic properties at the nanometer scale of supramolecular ureidopyrimidinone (UPy) based polymers in water. The UPy moiety is a selfcomplementary unit containing a quadruple hydrogen bonding array. In chloroform the high binding constant of $6 \times 10^{7} \mathrm{M}^{-1}$ results in a bonding life-time of roughly one second. ${ }^{16}$ In order to obtain similar values in water, the UPy unit requires hydrophobic shielding from water-soluble oligo- and poly(ethylene glycol) groups. ${ }^{17}$ Additionally, urea groups are introduced in the hydrophobic alkyl spacers to enable lateral stacking into long 1D-polymers due to a combination of hydrophobic effects, $\pi-\pi$ stacking and hydrogen bonding. Recently, we have shown that both mono- and bivalent UPy monomers assemble into supramolecular polymers that give rise to hydrogels at concentrations of only a few weight percentage (wt\%). ${ }^{17}$ By mixing the mono- and bivalent UPy monomers, hydrogels are obtained of which their stiffness can be controlled by the composition of the mixture. ${ }^{18}$

The structural and temporal properties of the assembly of monovalent monomers mUPy 1 and bivalent monomers bUPy 2 (Scheme 1) in water has been elucidated using cryogenic transmission electron microscopy (cryo-TEM), STORM and FRET experiments. For the latter two techniques, $2 \mathrm{~mol} \%$ of dye-labelled probes (monovalent mDye $\mathbf{3 a}$ and mDye $\mathbf{3 b}$, and bivalent bDye 4a and bDye $\mathbf{4 b}$; Scheme 1) were embedded in the aggregates formed by monomers 1 and 2 . 


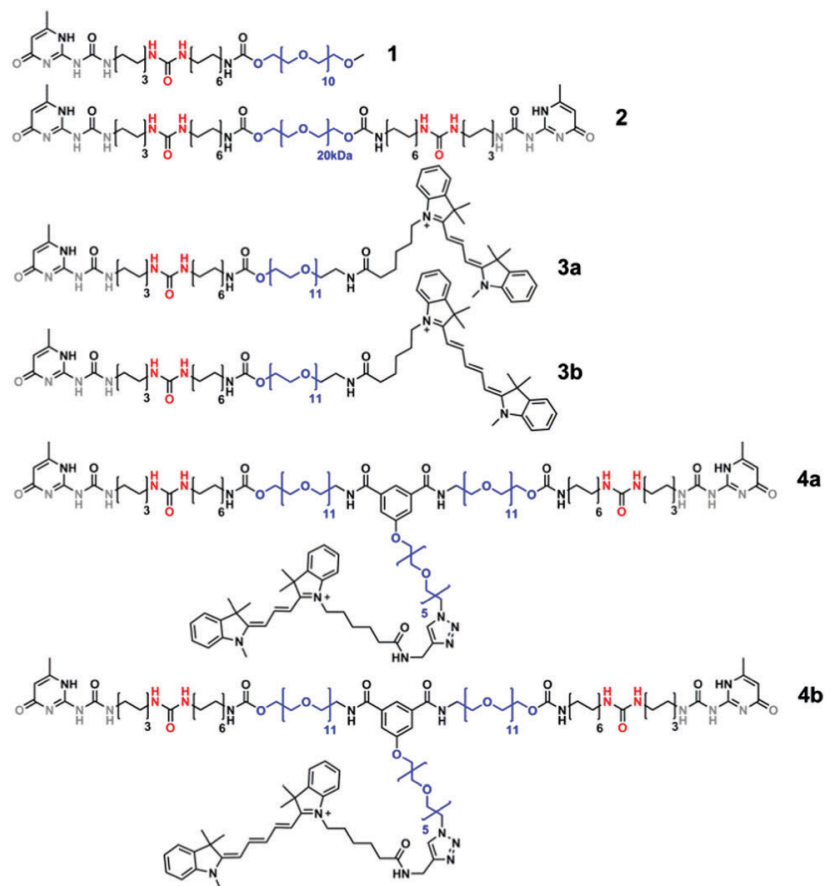

Scheme 1 Molecular structures of monovalent mUPy 1 , bivalent bUPy 2 dye-labelled monovalent UPy mDye $\mathbf{3 a}$ and mDye $\mathbf{3 b}$, and dye-labelled bivalent UPy bDye $\mathbf{4 a}$ and bDye $\mathbf{4 b}$. The scaffolding materials 1 and $\mathbf{2}$ are studied in combination with 2 mol\% of dyes 3 and $\mathbf{4}$ that serve as molecular probes. The UPy based molecules dimerize via quadruple hydrogen bonding arrays (grey) and laterally stack via the urea moieties (red). Poly(ethylene glycol) spacers (blue) provide water solubility.

Solutions of $\mathbf{1}$ and 2 were stirred in water at $72{ }^{\circ} \mathrm{C}$ for 1.5 hours, and were then slowly cooled to room temperature. Subsequently, the structural characteristics of the scaffolds formed by 1 and 2 were compared using cryo-TEM. Micrometre long fibrillar structures with diameters of approximately 5 and $14 \mathrm{~nm}$ were observed for 1 , while shorter fibrillar structures in the range of hundreds of nanometres with an apparent diameter of approximately $7 \mathrm{~nm}$ were found for 2 (Fig. 1 and ESI, $\dagger$ Fig. S1). The $14 \mathrm{~nm}$ diameter of 1 corresponds to bundled dimers, whereas the $5 \mathrm{~nm}$ of $\mathbf{1}$ and $7 \mathrm{~nm}$ of $\mathbf{2}$ are proposed to correspond
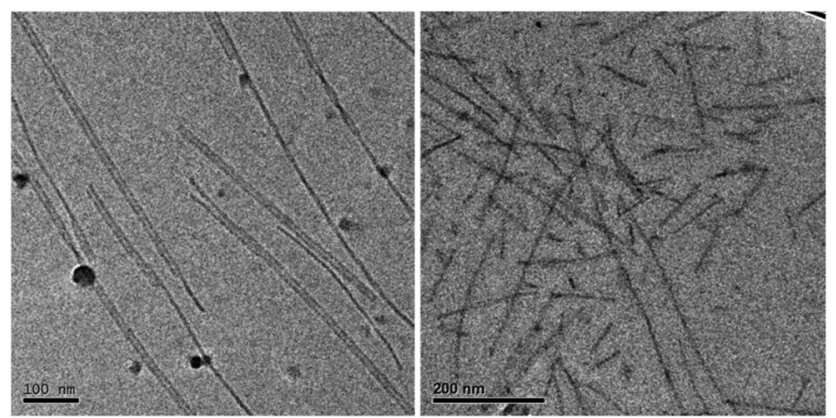

Fig. 1 Cryo-TEM images of scaffolds formed by monovalent mUPy 1 (left) and bivalent bUPy $\mathbf{2}$ (right). Fibrillar structures were observed in both cases with a diameter of approximately 5 and $14 \mathrm{~nm}$ for $\operatorname{mUPy} 1$ ( $c=0.5 \mathrm{mg} \mathrm{ml}^{-1}$; $482 \mu \mathrm{M})$ and approximately $7 \mathrm{~nm}$ for bUPy 2 ( $\left.c=10 \mathrm{mg} \mathrm{ml}^{-1} ; 474 \mu \mathrm{M}\right)$. The scale bars represent $100 \mathrm{~nm}$ (left) and $200 \mathrm{~nm}$ (right).
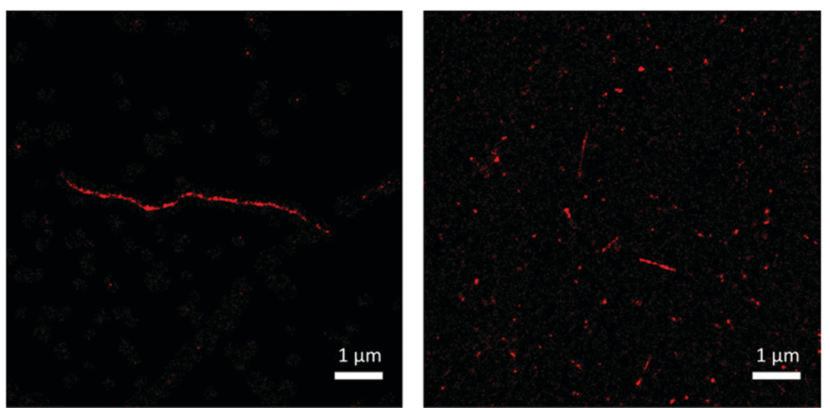

Fig. 2 STORM images of scaffolds formed by monovalent 1 (left) and bivalent 2 (right) with 2 mol\% of mDye $3 \mathbf{b}$ incorporated $\left(c_{\text {Total }}=12.5 \mu \mathrm{M}\right)$. Monovalent dye-labelled UPy $\mathbf{3 b}$ was incorporated into both scaffolds, showing micrometre long fibres for mUPy 1 and fibres of a few hundreds of nanometres for bUPy 2 . The scale bars represent $1 \mu \mathrm{m}$.

to single dimers. Please note that the water shell surrounding the PEG part of the molecules does not scatter sufficient electrons, and in the case of 2 , the longer PEG surrounding the fibre backbone sequesters more water, blurring the contrast of the inner hydrophobic part. The incorporation of the dye-labelled monomers $\mathbf{3 a}, \mathbf{3 b}, \mathbf{4 a}$, and $\mathbf{4 b}$ within these fibres was investigated using STORM. The measurements were performed at $12.5 \mu \mathrm{M}$ sample concentrations, using 1 or 2 in combination with $2 \mathrm{~mol} \%$ of the dye-labelled probes $\mathbf{3 a}, \mathbf{3 b}, \mathbf{4 a}$, or $\mathbf{4 b}$. Micrometre long fibre scaffolds were observed for $\mathbf{1}$ (with mDye $\mathbf{3 b}$, see Fig. 2, left), corroborating the cryo-TEM results for $\mathbf{1}$. In contrast, smaller, less-defined 1D-aggregates were observed for $\mathbf{2}$ (with mDye $\mathbf{3 b}$, see Fig. 2 right) with a maximal length of a few hundreds of nanometres. Despite the $>20$ fold poorer spatial resolution of the technique, ${ }^{19}$ the $\sim 40$ times lower concentration, and/or effects of sample preparation and adsorption on the glass surface, these structures formed by 2 appeared relatively similar as compared to the features observed using cryo-TEM. Nevertheless, the results of the two techniques showed a clear structural difference between the aggregates formed by the two UPy derivatives, where the long fibres of $\mathbf{1}$ and the shorter and lessdefined fibres of 2 indicate a differentiation in the packing of the monomers.

Next, the excellent FRET pair, donor Cy3 and acceptor Cy5, ${ }^{20}$ were selected for studying the exchange of monomeric units in and between 1D-fibre scaffolds. Separate solutions containing Cy3 (mDye 3a or bDye $\mathbf{4 a}$ ) and Cy5 (mDye $\mathbf{3 b}$ or bDye $\mathbf{4 b}$ ) were prepared by incorporating $2 \mathrm{~mol} \%$ of these dye-probes $\mathbf{3 a}, \mathbf{3 b}$, 4a or $4 \mathbf{b}$ into pre-assembled scaffold solutions containing 1 or 2 . Specifically, a $24.5 \mu \mathrm{M}$ scaffold and $0.5 \mu \mathrm{M}$ dye probe concentrations were used (sample preparation described in the ESI $\dagger$ ). Subsequently, the corresponding Cy3 and Cy5 solutions (i.e. $3 \mathbf{a}$ and $\mathbf{3 b}$, or $\mathbf{4 a}$ and $\mathbf{4 b}$ ) were mixed in a $1: 1$ ratio. Upon excitation of the donor, the fluorescence of both dyes was measured (Fig. 3). It is known that a close proximity $(2-10 \mathrm{~nm})$ of both dyes results in efficient energy transfer. ${ }^{8}$ Because of the non-covalent interactions between the monomers, the dyelabelled monomers can migrate within and between fibres. As a result, this technique investigates the exchange dynamics of the dye-labelled monomers within and between fibres. Two distinct 


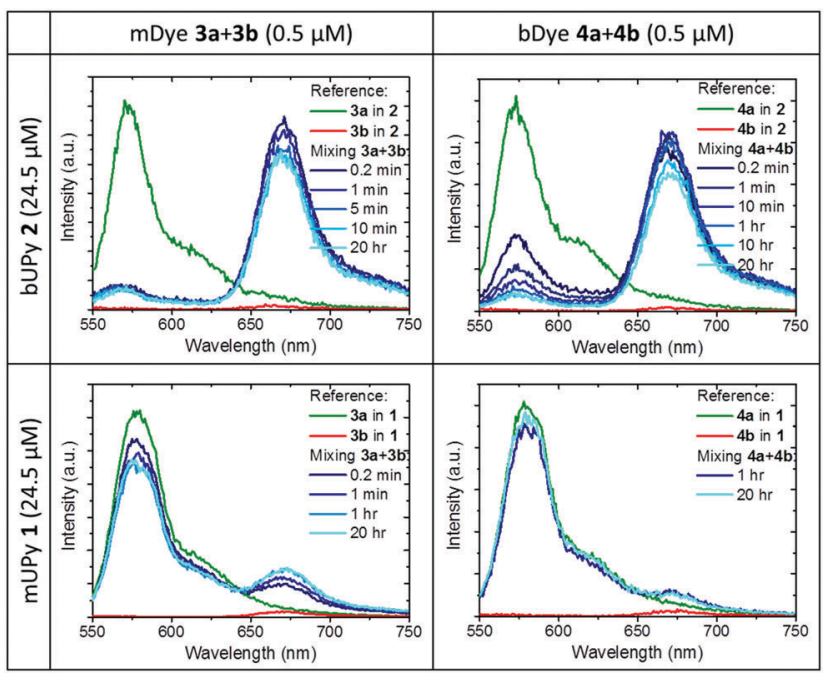

Fig. 3 Emission scans recorded after mixing solutions of mDye $3 \mathbf{a}$ and $\mathbf{3 b}$ or bDye $\mathbf{4 a}$ and $\mathbf{4 b}$ incorporated in scaffolds formed by mUPy $\mathbf{1}$ or bUPy $\mathbf{2}$ at different time points $\left(c_{\text {scaffold }}=24.5 \mu \mathrm{M}, c_{\text {Dye }}=0.5 \mu \mathrm{M}\right.$, excitation at $540 \mathrm{~nm}$ ). Immediate exchange occurred in samples containing bUPy 2 whereas a very low FRET was observed in samples containing mUPy 1.

exchange rates for the two scaffolds $\mathbf{1}$ and 2 were observed (Fig. 3 and ESI, $\dagger$ Fig. S2): immediately after mixing, a very efficient FRET was observed for mDyes $\mathbf{3 a}$ and $\mathbf{3 b}$ incorporated in 2 (Fig. 3, upper left), whereas only a low FRET was observed for mDyes $\mathbf{3 a}$ and $\mathbf{3 b}$ incorporated in $\mathbf{1}$ (Fig. 3, lower left). When increasing the temperature, the exchange of $\mathbf{1}$ was accelerated, even though the energy transfer did not reach a maximum, as was observed with 2 (ESI, $\dagger$ Fig. S3). Comparing the exchange rates of monovalent mDyes 3 and bivalent bDyes 4 within the same scaffold, a slower exchange profile for $\mathbf{4}$ was observed (Fig. 3, compare left and right). This is hypothesized to be due to multivalency effects. In addition, the exchange dynamics of 4 embedded in 2 were further reduced when the FRET experiments were performed with aged samples (ESI, $\dagger$ Fig. S4).

Finally, monovalent scaffold $\mathbf{1}$ and bivalent scaffold 2 were co-assembled in different ratios in order to investigate the effect of fibre composition on the exchange dynamics (Fig. 4 and ESI, $\dagger$ Fig. S5). Upon increasing the content of 2 with
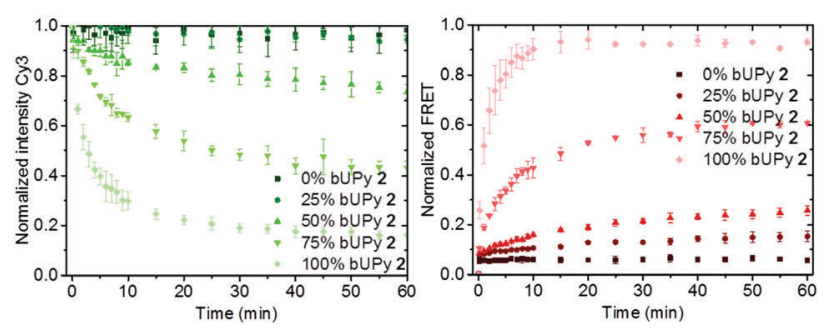

Fig. 4 Co-assembly of monovalent scaffold 1 with bivalent scaffold 2 , incorporated with 2 mol\% of bivalent dyes 4 ( $\left.c_{\text {Total }}=25 \mu \mathrm{M}\right)$. Left: Normalized Cy3 intensity; right: normalized intensity of the Cy5 peak upon Cy3 excitation, indicating FRET. Upon increasing the bivalent scaffold 2 content, the exchange dynamics were increased due to a disordering of the monomer packing. respect to $\mathbf{1}$, both the total exchange and the exchange rate were increased. The same trend was observed for both dyes, mDyes 3 and bDyes 4, however, a slightly lower FRET was observed with 4 when comparing the same fibre composition. Analysing the FRET experiments all-together, the dye-labelled monomers incorporated in $\mathbf{2}$ were more dynamic than in $\mathbf{1}$. Both the scaffold composition and the bivalency of the dyelabelled monomer influence the exchange dynamics. However, the scaffold composition dominates the kinetic properties, whereas the bivalency effect of the dye-labelled monomer is small. These observations indicate that the packing of the monomers is extremely important.

Since FRET is an ensemble technique and therefore only provides averaged data, the exchange dynamics of the dyelabelled monomers between individual fibres was visualized employing STORM. ${ }^{11}$ For this, the samples, as prepared for the FRET experiments, were diluted to $12.5 \mu \mathrm{M}$ with PBS and mixed in a $1: 1$ ratio with its corresponding variant (i.e. Cy3 solution a, $\mathbf{3 a}$ or $\mathbf{4 a}$, mixed with Cy5 solution $\mathbf{b}, \mathbf{3} \mathbf{b}$ or $\mathbf{4 b}$, respectively). The fibres were adsorbed on coverslips at different time points. Afterwards, dual channel imaging was performed where both Cy3 (green) and Cy5 (red) were excited simultaneously. Monovalent dyes $\mathbf{3 a}$ and $\mathbf{3 b}$ embedded in monovalent scaffold $\mathbf{1}$ after $\mathbf{1}$ minute of mixing ( $t=1 \mathrm{~min}$ ) revealed fibres which were predominantly green or predominantly red, indicating that negligible exchange had taken place (Fig. 5, upper row). However, after 20 hours, a

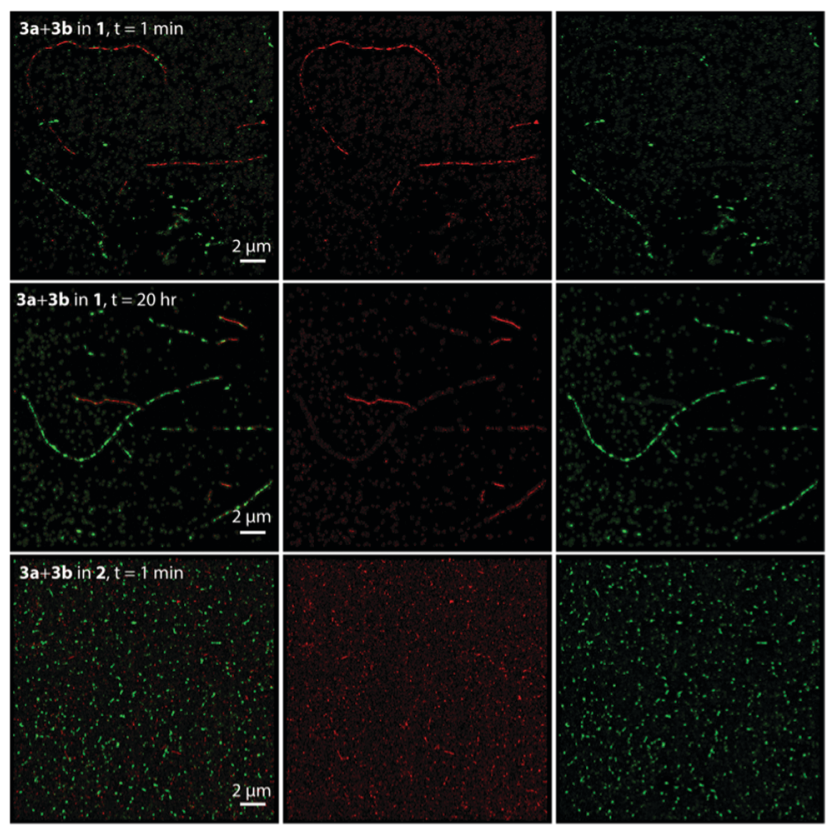

Fig. 5 STORM images of scaffolds formed by monovalent 1 (top and middle row) and bivalent 2 (bottom row) with 2 mol\% of incorporated mDye $3 \mathbf{a}+\mathbf{3 b}\left(c_{\text {Total }}=12.5 \mu \mathrm{M}\right)$. Left: Merged channel, middle: Cy5 excited channel, and right: Cy3 excited channel. Directly after mixing mDye $3 a$ and $\mathbf{3 b}$ incorporated in monovalent scaffold $\mathbf{1}$, negligible exchange was observed (top, $t=1 \mathrm{~min}$ ), and after 20 hours a small degree of exchange was observed (middle, $t=20$ hours). When the dyes were incorporated in bivalent scaffold 2 , instant exchange was observed (bottom, $t=1 \mathrm{~min}$ ). The scale bars represent $2 \mu \mathrm{m}$. 
small amount of random exchange was observed as a few red labels were observed in predominantly green fibres and vice versa. In contrast, exchange of the monovalent dyes $\mathbf{3 a}$ and $\mathbf{3} \mathbf{b}$ in 1D-fibers of bivalent scaffold 2 occurred immediately after mixing, indicated by both colours (i.e. green and red) being within the same fibre. Moreover, imaging of the mixed fibre (i.e. scaffolds containing $50 \% 1$ and $50 \%$ 2) showed fibres of intermediate lengths, demonstrating successful mixing and ruling out any selfsorting of 1 and 2 (ESI, $\dagger$ Fig. S6).

By using a combination of powerful techniques, the structural and temporal properties of UPy based supramolecular polymers were investigated on the molecular level. This revealed a clear difference between fibres formed by monovalent scaffold $\mathbf{1}$ and bivalent scaffold 2 . The long, well-defined fibres formed by 1 exhibited slow dynamics and even seemed to be near static. This is in sharp contrast with the structures formed by 2 , which showed only short fibres and proved to have a fast exchange profile. These results clearly show the impact of the molecular design on the microscopic structures that the molecules are forming. The large ethylene glycol chain in 2 ( $\sim 454$ vs. 10 repeating units in 1) destabilizes the self-assembly of the monomers due to steric and entropic effects, preventing an ordered packing and hence hampering the formation of well-defined fibres. The destabilization of supramolecular polymers by steric effects was also observed by Besenius et al. ${ }^{21}$ Furthermore, mixing of the UPy scaffolds formed by $\mathbf{1}$ and 2 resulted in destabilization of the ordered fibres upon an increasing content of 2 , which subsequently increased the exchange rate of the monomers. It is shown that the dynamics can be tuned by adjusting the fibre composition, predominantly dominated by a difference in packing. Mixing scaffold 1 in similar fibres as $\mathbf{2}$ was previously shown to improve the stiffness at the macroscopic level significantly. This was proposed to be due to a difference in exchange rates at the molecular level. ${ }^{18}$ Here we confirm that the exchange rates of the dye-labelled monomers are dominated by the scaffold type, and to a lesser extent by the multivalency of the dye-labelled monomer. Moreover, the hydrophobic nature of the dyes might result in a preference to reside in the hydrophobic pocket of the fibre. Although no clear differences in fibre formation were observed with cryo-TEM and UV-vis (ESI, $\dagger$ Fig. S7), changes in the exchange kinetics are likely to occur. $^{22}$

Despite it remaining a challenge to unravel the exact molecular structure and kinetics of supramolecular systems, cryoTEM, FRET and STORM proved to be a powerful combination of techniques to study supramolecular fibres in great detail. The results indicate that both the structure and dynamics of these fibres heavily depend on the design of the UPy monomers, and we showed that it is possible to control their properties by mixing the different monomer variants. These are important findings for the design of functional monomers such as bioactive peptides and proteins introduced into supramolecular fibres, since they might change the packing within the fibres, and as a consequence, the macroscopic properties. Because there is a delicate balance between dynamic spatiotemporal presentation and the ability of the cell to locally remodel the surrounding microscopic environment, we anticipate that the studies described here are useful for the design of biomaterials.

This work was supported by the European Research Council (Grant Agreement 246829), the Royal Netherlands Academy of Science, the Netherlands Organization of Sciences (NWO-Newpol), and the Dutch Ministry of Education, Culture and Science (Gravity program 024.001.035).

\section{Notes and references}

1 E. A. Appel, J. del Barrio, X. J. Loh and O. A. Scherman, Chem. Soc. Rev., 2012, 41, 6195-6214.

2 M. J. Webber, E. A. Appel, E. W. Meijer and R. Langer, Nat. Mater., 2016, 15, 13-26.

3 E. Krieg, M. M. C. Bastings, P. Besenius and B. Rybtchinski, Chem. Rev., 2016, 116, 2414-2477.

4 H. J. Anderson, J. K. Sahoo, R. V. Ulijn and M. J. Dalby, Front. Bioeng. Biotechnol., 2016, 4, 38.

5 M. Akhmanova, E. Osidak, S. Domogatsky, S. Rodin and A. Domogatskaya, Stem Cells International, 2015, 2015, 35.

6 G. Yu, X. Yan, C. Han and F. Huang, Chem. Soc. Rev., 2013, 42, 6697-6722.

7 A. K. Kenworthy, Methods, 2001, 24, 289-296.

8 N. D. Huebsch and D. J. Mooney, Biomaterials, 2007, 28, 2424-2437.

9 S. W. A. Reulen and M. Merkx, Bioconjugate Chem., 2010, 21, 860-866.

10 N. Momin, S. Lee, A. K. Gadok, D. J. Busch, G. D. Bachand, C. C. Hayden, J. C. Stachowiak and D. Y. Sasaki, Soft Matter, 2015, 11, $3241-3250$.

11 L. Albertazzi, D. van der Zwaag, C. M. A. Leenders, R. Fitzner, R. W. van der Hofstad and E. W. Meijer, Science, 2014, 344, 491-495.

12 R. M. P. da Silva, D. van der Zwaag, L. Albertazzi, S. S. Lee, E. W. Meijer and S. I. Stupp, Nat. Commun., 2016, 7, 11561.

13 S. Onogi, H. Shigemitsu, T. Yoshii, T. Tanida, M. Ikeda, R. Kubota and I. Hamachi, Nat. Chem., 2016, 8, 743-752.

14 E. A. Appel, R. A. Forster, A. Koutsioubas, C. Toprakcioglu and O. A. Scherman, Angew. Chem., Int. Ed., 2014, 53, 10038-10043.

15 E. T. Pashuck, H. Cui and S. I. Stupp, J. Am. Chem. Soc., 2010, 132, 6041-6046.

16 S. H. M. Söntjens, R. P. Sijbesma, M. H. P. van Genderen and E. W. Meijer, J. Am. Chem. Soc., 2000, 122, 7487-7493.

17 P. Y. W. Dankers, T. M. Hermans, T. W. Baughman, Y. Kamikawa, R. E. Kieltyka, M. M. C. Bastings, H. M. Janssen, N. A. J. M. Sommerdijk, A. Larsen, M. J. A. van Luyn, A. W. Bosman, E. R. Popa, G. Fytas and E. W. Meijer, Adv. Mater., 2012, 24, 2703-2709.

18 R. E. Kieltyka, A. C. H. Pape, L. Albertazzi, Y. Nakano, M. M. C. Bastings, I. K. Voets, P. Y. W. Dankers and E. W. Meijer, J. Am. Chem. Soc., 2013, 135, 11159-11164.

19 B. Huang, M. Bates and X. Zhuang, Annu. Rev. Biochem., 2009, 78, 993-1016.

20 R. B. Mujumdar, L. A. Ernst, S. R. Mujumdar, C. J. Lewis and A. S. Waggoner, Bioconjugate Chem., 1993, 4, 105-111.

21 R. Appel, J. Fuchs, S. M. Tyrrell, P. A. Korevaar, M. C. A. Stuart, I. K. Voets, M. Schönhoff and P. Besenius, Chem. - Eur. J., 2015, 21, 19257-19264.

22 M. B. Baker, R. P. J. Gosens, L. Albertazzi, N. M. Matsumoto, A. R. A. Palmans and E. W. Meijer, ChemBioChem, 2016, 17, 207-213. 